\title{
Strain of experimental animals and modulation of nitric oxide pathway: their influence on development of renal failure in an experimental model of hepatorenal syndrome
}

\author{
Marek Saracyn ${ }^{1}$, Janusz Patera ${ }^{2}$, Janusz Kocik³ ${ }^{3}$ Marek Brytan ${ }^{4}$, Robert Zdanowski ${ }^{4}$, Arkadiusz Lubas ${ }^{1}$, \\ Wojciech Kozłowski², Zofia Wańkowicz ${ }^{1}$
}

\author{
1Department of Internal Diseases, Nephrology and Dialysis, Military Institute of Health \\ Services, Warsaw, Poland \\ 2Department of Pathology, Military Institute of Health Services, Warsaw, Poland \\ ${ }^{3}$ Military Institute of Hygiene and Epidemiology, Warsaw, Poland \\ 4Department of Pharmacology and Toxicology, Military Institute of Hygiene and \\ Epidemiology, Warsaw, Poland
}

Submitted: 19 April 2011

Accepted: 20 December 2011

Arch Med Sci 2012; 8, 3: 555-562

DOI: 10.5114/aoms.2012.29281

Copyright (c) 2012 Termedia \& Banach

\section{Abstract}

Introduction: Pathomechanism of HRS is still poorly understood. The aim of our study was: (1) to test whether different strains of rats could develop typical HRS, and (2) to estimate the influence of activation and inhibition of nitric oxide for development of renal failure in course of HRS.

Material and methods: First, we used 16 of Wistar and 16 of Sprague-Dawley rats in galactosamine model of HRS. Next, we used 48 of SDR rats, which received saline, N-nitro-L-arginine or L-arginine before and after liver damage. Twenty four hours urine and blood samples were collected $48 \mathrm{~h}$ after saline or Ga1N injection. Biochemical parameters were determined in serum or urine and then creatinine clearance and osmolality clearance were calculated. Liver and kidney tissues were collected for histopathological examination.

Results: Liver failure developed in all tested groups with significant increase of bilirubin $(p<0.001)$, ALT $(p<0.001)$ and ammonia $(p<0.001)$. Nevertheless we did not achieve any evidence of renal failure in Wistar, but we found typical renal failure in Sprague-Dawley group with significant decrease in creatinine clearance $(p<0.0012)$ and increase in concentration of creatinine and urea $(p<0.001)$ and $(p<0.001)$ respectively. Inhibition of NOS prevented development of renal failure with significant improvement of GFR both before $(p<0.0017)$ and after $(p<0.003)$ Ga1N injection. Injection of L-arginine after Ga1N injection did not caused significant improvement of GFR.

Conclusions: Our study showed, that genetic factors might be responsible for development of renal failure in course of HRS and nitric oxide play important role in acute model of this syndrome.

Key words: hepatorenal syndrome, experimental studies, nitric oxide.

\section{Introduction}

Hepatorenal syndrome (HRS) remains one of the few great mysteries of medicine. The illness has a severe clinical course, poor prognosis and high mortality. According to the definition accepted in 1994 by the Inter-

\author{
Corresponding author: \\ Marek Saracyn MD, PhD \\ Department of Internal \\ Diseases, Nephrology \\ and Dialysis \\ Military Institute \\ of Health Services \\ 128 Szaserów \\ 04-141 Warsaw, Poland \\ Phone: + 48226816811 \\ Fax: + 48226816811 \\ E-mail:msaracyn@interia.pl
}


national Ascites Club, developed in 1996 and redefined in 2007 by expert consensus, HRS is a functional renal failure appearing in the course of severe liver damage and liver failure [1-3]. Liver disease is the only etiological factor and specific acute renal failure (ARF) characterised by hyperazotemia, oliguria and hyponatremia develops without clinical, laboratory and histological features of any common kidney disease [4, 5]. In clinical practice, HRS usually develops in liver cirrhosis, acute hepatitis, and primary biliary cirrhosis. However, up to now contemporary medicine has not found an effective treatment of this syndrome, generally because precise pathomechanisms leading from hepatic damage to renal failure are still very poorly understood [6].

The hypothesis of arterial vasodilation is a wellknown and universally accepted theory of how renal failure develops after liver damage. According to this hypothesis, damage of the liver leads to vasodilation of the splanchnic vascular bed, then to specific underfilling in the systemic circulation and by activating vascular baroreceptors to extreme stimulation of systemic vasoconstrictor factors, e.g. the renin-angiotensin system, sympathetic nervous system or arginine-vasopressin system. The whole process results in vasoconstriction of the renal vascular bed and hypoperfusion of the kidney $[7,8]$. As some studies have shown, splanchnic vessels are not affected by systemic vasoconstrictors, chiefly because of vasodilator factors produced locally. The main role in these mechanisms would be played by nitric oxide [9-12].

In the case of acute liver damage precise pathomechanisms of HRS are less well known. As some studies have shown, liver damage in a short time leads to portal hypertension, increase of portal inflow as a consequence of opening collateral vessels and to vasodilation of the splanchnic vascular bed. This vasodilation of the splanchnic vascular bed initiates the cascade of biological phenomena described above $[13,14]$. The role of the nitric oxide pathway has been well established hitherto in chronic models of HRS, especially in cirrhosis $[15,16]$. There are no such solid data on the function of the endogenous NO system in the acute model of liver and renal failure. To test the pathophysiological impact of NO in different biological systems, experimental studies use, on one hand, inhibitors of that system, such as nitric oxide synthase inhibitors (e.g. $N$-nitroL-arginine - L-NAME), and on the other, NO donors, such as L-arginine.

There are a few reported experimental models of HRS, e.g. the carbon tetrachloride intoxication model, and the bile duct ligation model [17-19], but one of the most reproducible and widely acceptable is a rat galactosamine intoxication model, induced by intraperitoneal injection of galactosamine [20, 21]. Galactosamine (Ga1N) injected intraperitoneally in rats causes acute liver failure and subsequently development of acute functional renal failure. As previously shown, renal failure develops typically after liver damage in male Sprague-Dawley rats [22]. There are no data on the typical HRS induced by Ga1N in other experimental strains of rats. It is worth considering whether other strains develop acute functional renal failure after liver damage in the same way, in other words, whether some inborn constitutional factors determining development of acute HRS really exist. Furthermore, we know from clinical practice that there are some patients both with HRS and also with other types of kidney diseases (e.g. diabetic, atherosclerotic, ischaemic, etc.) in whom renal failure progresses slower or faster than in others. Therefore, finding animals with different rates of renal failure development, or not developing ARF at all, could uncover protective factors against the progression of renal failure in this syndrome.

The aim of our study was: (1) to test whether different strains of rats could develop typical hepatorenal syndrome in the same way, and (2) to assess the influence of activation and inhibition of the nitric oxide system on development and degree of renal failure in the course of acute liver damage.

\section{Material and methods}

In the experiments we generally used randomly selected 24 male Wistar (WR) and 64 male SpragueDawley (SDR) rats, of body weight 200-250 g, obtained from the Department of Experimental Animals of the Polish Mother's Memorial Hospital in Lodz. In particular: (1) in the first part of the experiment (groups 1-5) we used 16 individuals of the WR and 16 of the SDR rats (groups 1, 2 and 4, 5 respectively, 8 individuals in each tested group), and the additional group 3 of WR $(n=8)$, with double dose of galactosamine ( $2 \times G a 1 N / W R)$, because a single dose of Ga1N (group 2, $n=8,1 \times$ Ga1N/WR) failed to develop HRS in WR rats (group 3 vs. group $1-W R$ vs. $W R$ ); (2) in the second part of the study (groups 6-11), we used 48 SDR rats, as susceptible to HRS, which were given saline, L-NAME or L-arginine injection.

The animals were kept in standard group cages and fed a standard diet, with free access to water and food, with a natural day/night cycle of $12 \mathrm{~h}$, at a temperature of $22 \pm 2^{\circ} \mathrm{C}$, and humidity of $45-50 \%$. The experiments were performed from $10.00 \mathrm{a} . \mathrm{m}$. to 6.00 p.m. on natural moving animals in their waking time. The studies were carried out according to the guidelines of Animals in Scientific Procedures. In the course of experiments rats were placed individually in glass metabolic cages with free access to water and food. Animals were divided into eleven groups, eight individuals in each: group 1 - control WR group - received $1 \mathrm{ml}$ of $0.9 \%$ saline solution intraperitoneally (i.p.); group 2 - tested WR group - received $1.1 \mathrm{~g} / \mathrm{kg}$ b.w. of galactosamine hydrochlo- 
ride (Ga1N - Sigma Aldrich Polska) via intraperitoneal injection as a $200 \mathrm{mg} / \mathrm{ml}$ solution in $0.9 \%$ saline; group 3 - tested WR group - was given $2.2 \mathrm{~g} / \mathrm{kg}$ b.w. of galactosamine hydrochloride injected i.p. as a $200 \mathrm{mg} / \mathrm{ml}$ solution in $0.9 \%$ saline; group 4 - control SDR group - received $1 \mathrm{ml}$ of $0.9 \%$ saline solution i.p.; and group 5 - tested SDR group - was given $1.1 \mathrm{~g} / \mathrm{kg}$ b.w. of galactosamine hydrochloride via intraperitoneal injection as a $200 \mathrm{mg} / \mathrm{ml}$ solution in $0.9 \%$ saline. Group 6 - SDR, control L-NAME group - received i.p. $100 \mathrm{mg} / \mathrm{kg} N$-nitro-L-arginine (L-NAME - Sigma Aldrich, Polska); group 7 - SDR, the same dose of L-NAME 48 and $24 \mathrm{~h}$ before Ga1N injection; group 8-SDR, the same dose of L-NAME 24 and $48 \mathrm{~h}$ after Ga1N injection. Group 9 - SDR, control L-ARG group, received i.p. $150 \mathrm{mg} / \mathrm{kg}$ b.w. L-arginine (L-ARG - Sigma Aldrich Polska); group 10 - SDR, received the same dose of L-ARG 48 and $24 \mathrm{~h}$ before Ga1N injection; group 11 - SDR, the same doses of L-ARG 24 and $48 \mathrm{~h}$ after Ga1N injection.

Twenty-four-hours urine samples were collected during $24 \mathrm{~h}$ from the $24^{\text {th }}$ to $48^{\text {th }} \mathrm{h}$ after saline or Ga1N injection and evaluated $48 \mathrm{~h}$ after saline or Ga1N injection. Blood samples of $6 \mathrm{ml}$ were also collected $48 \mathrm{~h}$ after saline or Ga1N injection from the beating heart in deeply anaesthetised animals. Biochemical parameters, except ammonium, were determined in serum or urine by means of an Integra 700 autoanalyser (Roche, USA), Bilirubin, ALT, AST, Creatinine, Urea, Sodium Reagents (Roche, Germany); and ammonium concentration in plasma with EDTA-K 3 anticoagulant by an autoanalyser. Urine osmolality was measured by means of an automatic osmometer (Osmometer Automatic, Knauer, Germany) (Anand et al., 2002). Creatinine clearance as a parameter of glomerular filtration was calculated according to the equation: creatinine clearance $[\mathrm{ml} / \mathrm{min}]=\left(\right.$ Creat $_{u} \times$ $\left.\mathrm{V}_{\mathrm{u} 24 \mathrm{~h}}\right) /\left(\right.$ Creat $\left._{\mathrm{s}} \times 1440\right)$, where: Creat $_{u}-$ urine creatinine, Creat $_{\mathrm{s}}$ - serum creatinine, $\mathrm{V}_{\mathrm{U} 24 \mathrm{~h}}-24 \mathrm{~h}$ urine volume. Osmolality clearance was calculated according to the equation: osmolality clearance [ml/day] $=\left(\right.$ Uosm $\left.\times V_{U 24 h}\right) /$ Posm, where: Uosm - urine osmolality, $V_{U} 24 \mathrm{~h}-24 \mathrm{~h}$ urine volume, Posm plasma osmolality, calculated according to the equation: $2 \times\left(\mathrm{Na}_{\mathrm{s}}+\mathrm{K}_{\mathrm{s}}+\right.$ Urea $\left._{\mathrm{s}}\right)$, where: $\mathrm{Na}_{\mathrm{s}}-$ serum sodium, $\mathrm{K}_{\mathrm{s}}$ - serum potassium, Urea $\mathrm{s}_{\mathrm{s}}$ - serum urea [22].

After exsanguination, liver and kidney tissues were collected for histopathological examination: liver and kidney sections were fixed in formalin, paraffin embedded, stained with haematoxylin and eosin and examined in light microscope.

\section{Statistical analysis}

Statistical analysis was performed using Student's t-test and ANOVA when multiple comparisons were required. Where appropriate, the Mann-Whitney test was used to analyse non-parametric data. The limit of significance was taken as $p<0.05$. All data are expressed as means $\pm \mathrm{SE}$.

\section{Results}

Table I shows liver and renal parameters obtained from WR and SDR groups. Galactosamine intoxication caused damage of liver and subsequently liver failure in all groups, both in WR and SDR rats (groups 2, 3 and 5) with significant increase in serum concentration of bilirubin, ALT and ammonia in comparison to control groups (group 1 and 4 respectively). In group 2 (WR), given $1.1 \mathrm{~g} / \mathrm{kg}$ Ga1N, we found a significant increase of serum bilirubin $(p<0.001)$, ALT $(p<0.001)$ and ammonia $(p<0.001)$ in comparison to the sham group (group 1) (Table I). However, we did not observe any evidence of acute renal failure in group 2 in the biochemical profile used in our study. There were not any typical differences of acute renal failure in concentration of serum creatinine and urea, in creatinine clearance or urine osmolality. Some parameters behaved completely differently from what might be expected in acute functional renal failure typical of HRS, e.g. concentration of urine sodium in group 2 increased significantly $(p<0.002)$.

In group $3(\mathrm{WR})$, given $2.2 \mathrm{~g} / \mathrm{kg}$ Ga1N, liver injury and liver failure also developed after $48 \mathrm{~h}$. The degree of liver failure was higher than in group 2 and characterised by significant increase of serum bilirubin level $(p<0.002)$, ALT $(p<0.003)$ and ammonia $(p<0.001)$ in the sham and tested group 3 respectively (Table I). Therefore, the degree of liver failure was dependent on the dose of galactosamine. Nevertheless, in this WR tested group, we also failed to find any evidence of acute renal failure in biochemical profiles of kidney function used in our study.

However, in the tested Sprague-Dawley group (group 5) we found biochemical parameters of acute renal failure after hepatic damage typical of HRS with a significant decrease in creatinine clearance and increase in serum concentration of creatinine and urea (Table I, Figures 1, 2) In comparison to the control (group 4), in an SDR tested group (group 5), creatinine clearance decreased significantly $(p<0.0012)$, while concentration of creatinine and urea increased, also significantly $(p<0.001$ and $p<0.001$ respectively). There were no significant differences between the control SDR group (group 4) and control L-NAME group (group 6).

In group 7, given L-NAME before Ga1N intoxication, we found significant improvement of GFR $(p<0.0017)$ (Figure 1) and a significant decrease of serum creatinine $(p<0.001)$ and urea $(p<0.001)$ concentration. The 24-h urine volume and osmolar clearance increased significantly $(p<0.0005$ and $p<0.023$ respectively). Inhibition of the NO system after Ga1N intoxication (group 8 - given L-NAME after Ga1N) also significantly increased GFR $(p<0.003)$ 


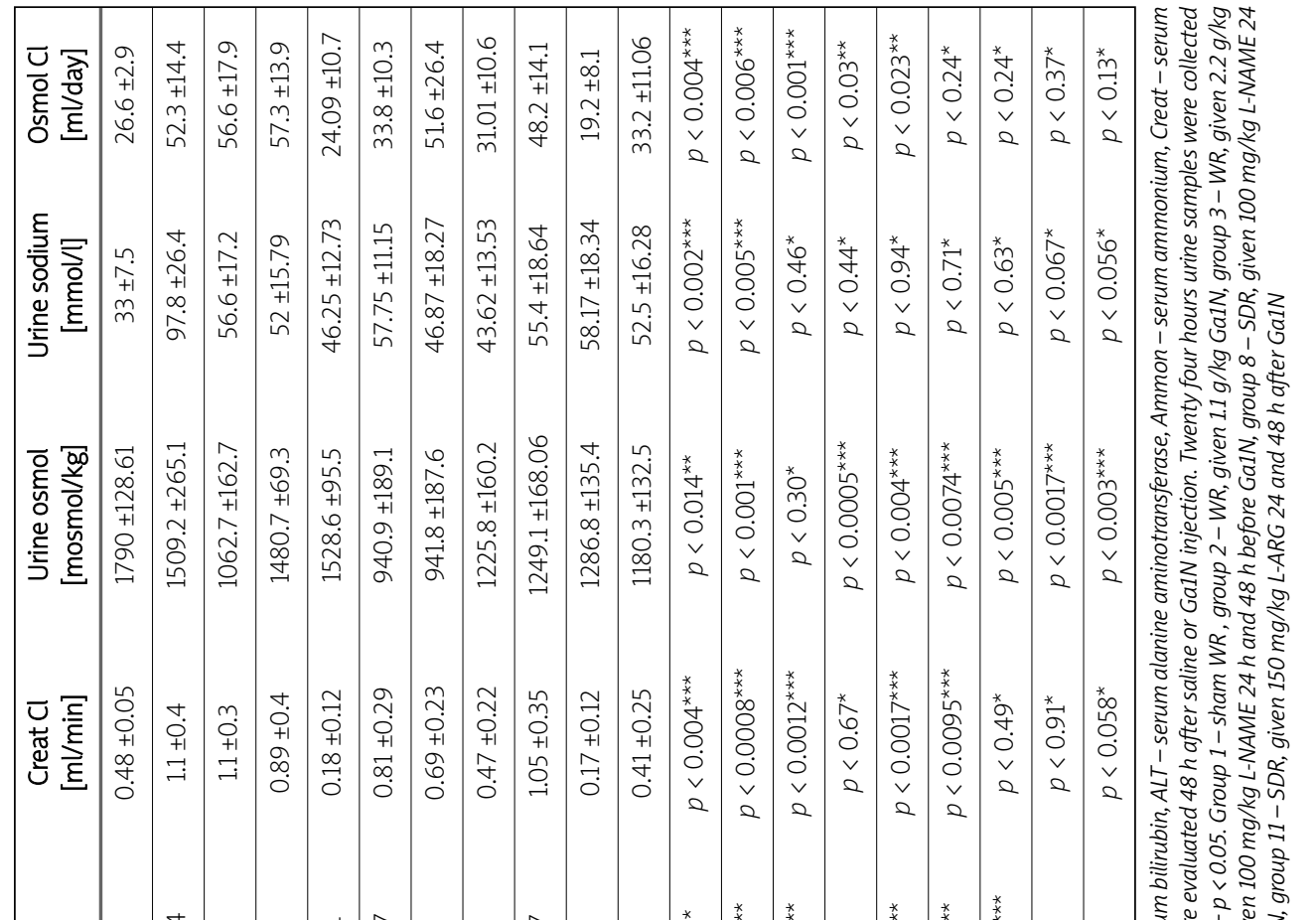

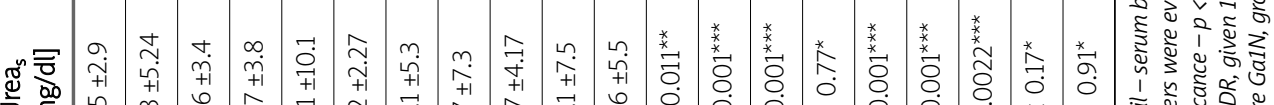

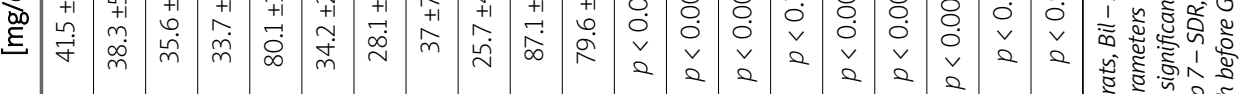

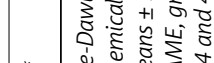

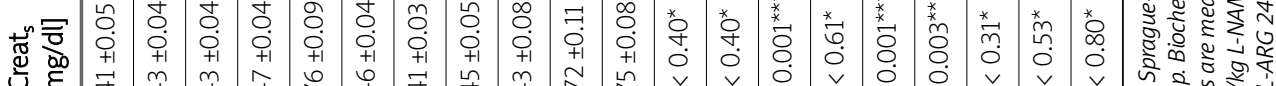

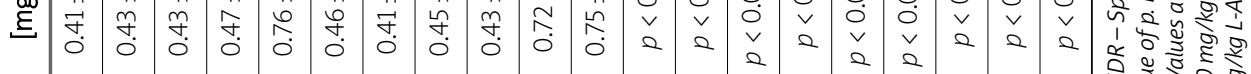

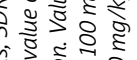

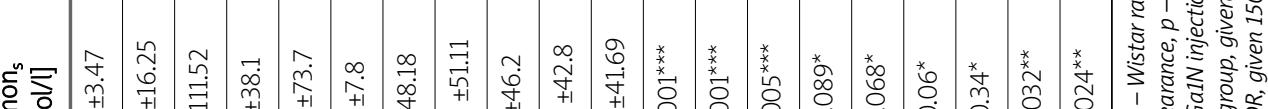

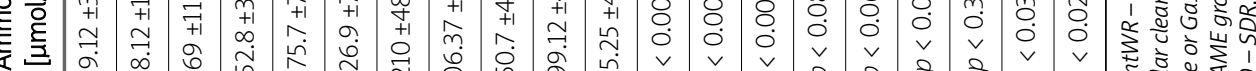

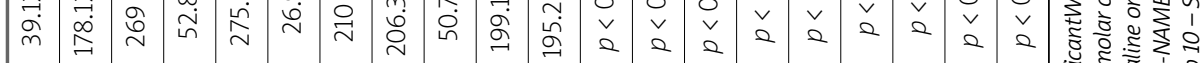

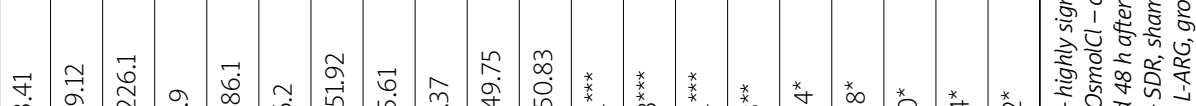

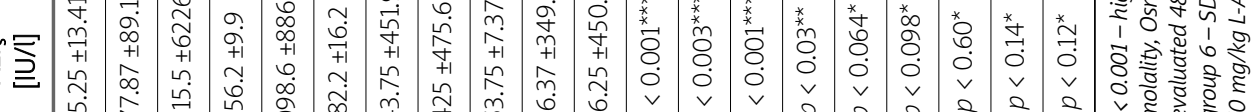

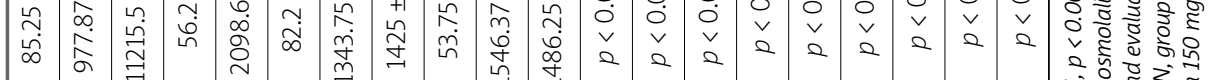

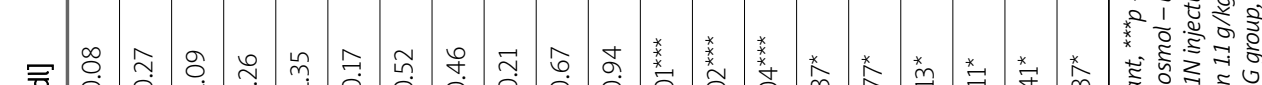

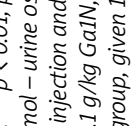

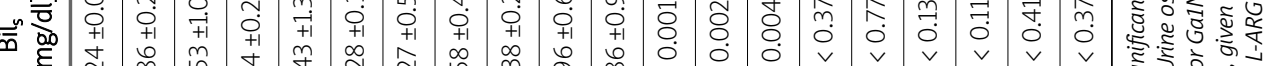

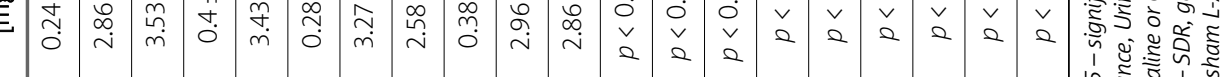

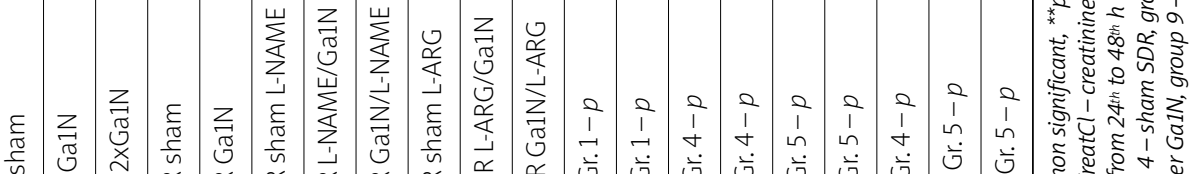

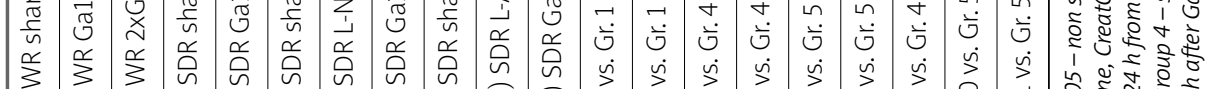

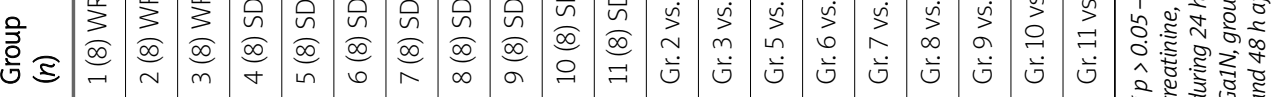




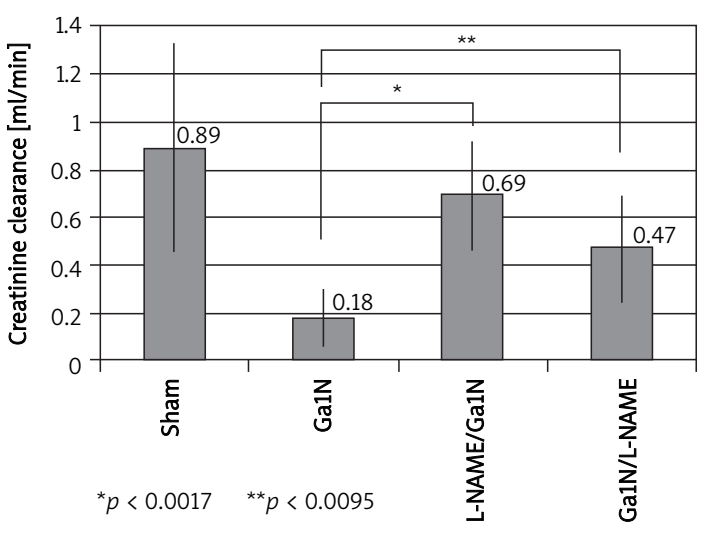

Figure 1. Influence of L-NAME injection on creatinine clearance before and after liver injury. Inhibition of NO system before Ga1N intoxication significantly increased GFR. Inhibition of NOS after Ga1N intoxication also resulted in significant improvement of GFR, but to a lesser degree than in the case of treatment before intoxication. Values are means $\pm \mathrm{SE}$, significance $-p<0.05$

(Figure 1), but to a lesser degree than in the previous group. Serum concentration of creatinine and urea decreased significantly $(p<0.003$ and $p<0.001$ respectively) in comparison to the Ga1N group (group 5). There were no changes in osmolar clearance in this group. Nor were there any significant changes between the control SDR group (group 4) and the control L-ARG group (group 9).

In the tested group 10, given L-ARG before Ga1N intoxication, we did not find any significant changes in GFR (Figure 2), serum concentration of creatinine, urea, osmolar clearance and 24-h urine volume. An attempt to activate the NO system after Ga1N injection (group 11, given L-ARG after Ga1N) caused a considerable and almost significant increase of GFR ( $p<0.058$ ) (Figure 2) and increase of 24-h urine volume $(p<0.006)$ in comparison to the Ga1N group (group 5).

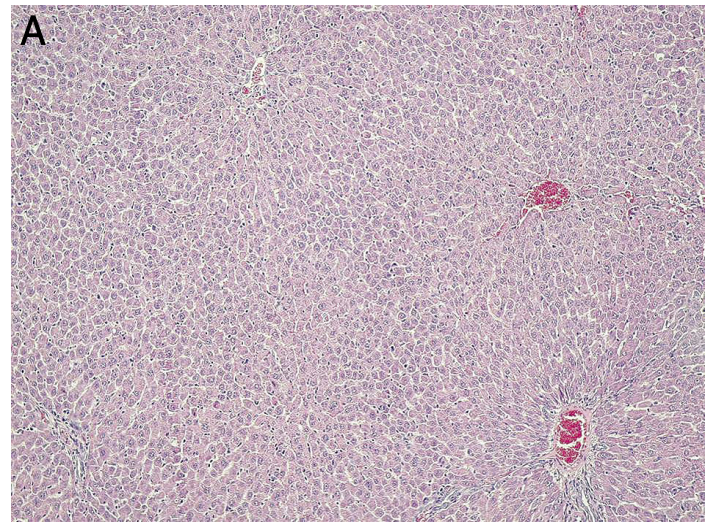

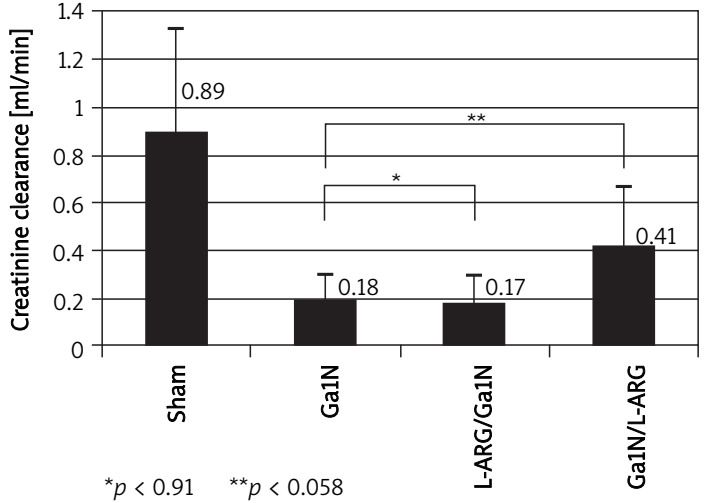

Figure 2. Influence of L-ARG injection on creatinine clearance before and after liver injury. Activation before liver damage did not significantly change level of GFR. However, using a donor for the NO system after Ga1N intoxication brought improvement of glomerular filtration, although not significantly. Values are means $\pm \mathrm{SE}$, significance $-p<0.05$

A histological examination of liver tissue (Figure 3) confirmed the development of massive necrosis of hepatocytes in all tested groups (groups 2, 3, 5, 7, 8, 10 and 11) in contrast to the control groups (groups 1 , 4, 6 and 9), where no abnormalities were found. A histological examination of kidney (Figure 4) tissue showed no changes in any of the tested or control groups.

\section{Discussion}

Clinical studies of HRS sometimes encounter various objective difficulties. Some chemical compounds which are necessary for such studies are often not permitted for tests on human populations. Moreover, clinical studies of HRS usually refer to small groups, difficult to compare, in view of the large variety of etiological factors, and different duration and severity of the disease. In this situation experimental studies give objective results, because they

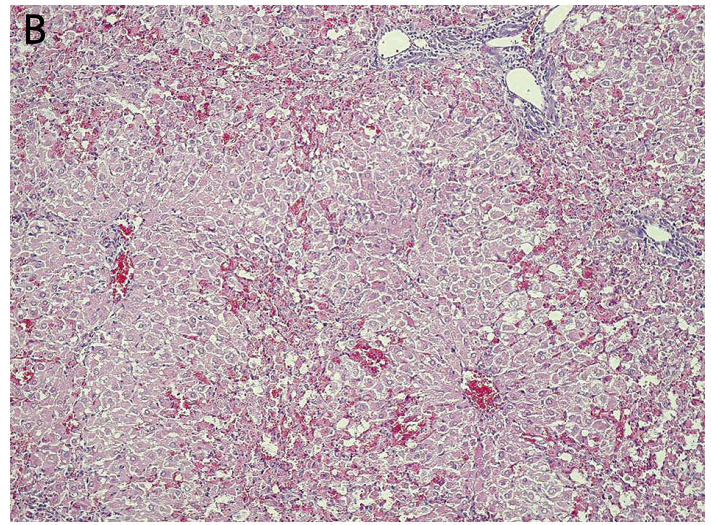

Figure 3. Histopathological picture of the liver in SDR. A - Lack of morphological changes in the liver from sham SDR rats (group 4) at $48 \mathrm{~h}$ after saline injection. Haematoxilin and eosin staining, light microscope, magnification 10x. B - Massive necrosis of hepatocytes in the liver from tested SDR rats (group 5) at $48 \mathrm{~h}$ after galactosamine injection. Haematoxilin and eosin staining, light microscope, magnification 10x 

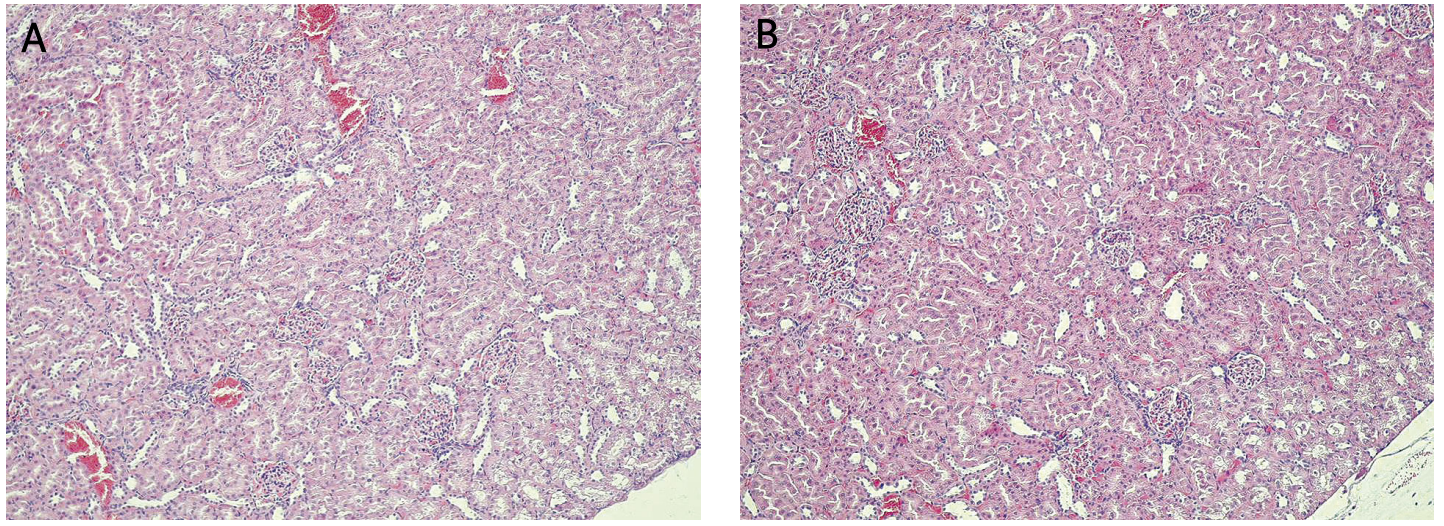

Figure 4. Histopathological picture of the kidney in SDR. A - Lack of morphological changes in the kidney from sham SDR rats (group 4) at $48 \mathrm{~h}$ after saline injection. Hematoxilin and eosin staining, light microscope, magnification 10x. B - Lack of morphological changes in the kidney from tested SDR rats (group 5) at $48 \mathrm{~h}$ after galactosamine injection. Hematoxilin and eosin staining, light microscope, magnification 10x

test individuals of the same strain, age, gender, body weight and in the same experimental conditions. At the same time it is possible to use the whole tested material: blood, urine and tissue slices. Hence the results are more reliable and objective. Consequently, experimental studies become not only a supplement of clinical studies, but sometimes the only way to explain some biological phenomena.

Among a few experimental models of HRS, we used an acute rat model, induced by intraperitoneal intoxication with galactosamine [17-21]. Galactosamine (Ga1N) injected intraperitoneally usually at the dose of $1.1 \mathrm{~g} / \mathrm{kg}$ within $48 \mathrm{~h}$ after intoxication induces in rats acute hepatic injury and liver failure and subsequently acute functional renal failure. Liver failure is characterized by a large increase of serum bilirubin, ALT, AST concentration, decrease of albumin concentration, and histologically, massive necrosis of hepatocytes. In the biochemical profile of renal function an increase of serum concentration of creatinine and urea is found with parallel decrease of renal blood flow and glomerular filtration rate caused by renal vasoconstriction. Mean arterial pressure is lower than in sham rats. Because of peripheral underfilling and diminished vascular resistance, cardiac output increases and leads to hyperdynamic circulation.

In the literature to date, only Anand et al. have established a standardized and reproducible model of acute functional renal failure as a consequence of acute liver failure in Sprague-Dawley rats after Ga1N intoxication [22]. But there are no data on the typical HRS induced by Ga1N in other experimental strains of rats.

Therefore, in our study we compared Wistar and Sprague-Dawley rats. In the Wistar, unlike the Sprague-Dawley group, despite univocal biochemical and histological parameters of hepatic injury and development of acute liver failure, we did not achieve secondary acute renal failure, although biochemical parameters and the histological picture of liver failure were comparable with those obtained in the Sprague-Dawley group. Moreover, in tested group 3, given $2.2 \mathrm{~g} / \mathrm{kg}$ of Ga1N, the degree of liver failure was clearly higher than in Wistar group 2 and SpragueDawley group 5, both given $1.1 \mathrm{~g} / \mathrm{kg}$ of Ga1N. Therefore in spite of more severe hepatic damage, it remained without any influence on renal function. Javle et al. [23] used Wistar rats in their studies, but the aim of their work was primarily splanchnic haemodynamic changes, without any analysis of renal function. Erdely et al. [24] in a model of 5/6 nephrectomy showed that renal synthesis of NO was higher and the number of working nephrons was about 30\% greater in Wistar rats as compared with SpragueDawley rats. This protective mechanism of NO was also confirmed in other models of chronic kidney disease [25-27]. On the other hand, so far no studies have been reported comparing WR and SDR animals in an acute model of HRS. So, we speculate that the main difference in resistance to development and progression of renal failure in this acute hepatorenal syndrome results from the existence of some protective vasoactive factors. Local renal vasodilators, such as prostaglandins, the kallikreinkinin system, natriuretic peptides or the local nitric oxide system, could be good candidates. Moreover, a high number of active nephrons is also a widely acceptable nephroprotective factor, and a smaller number of such nephrons in the case of SDR is probably also one of the determining factors of progression of renal failure. So, further studies are necessary to evaluate the nephroprotective factors mentioned above in an acute model of HRS.

Therefore the strain of experimental animals is probably one of the factors responsible for the development of acute renal failure in the course of experimental acute liver failure. Our study may prove 
that genetic factors play an important role in pathogenesis of the experimental hepatorenal syndrome.

In our study in an acute experimental model of HRS, the inhibition of the NO system before Ga1N intoxication significantly increased GFR and decreased concentration of creatinine and urea. On the other hand, the inhibition of NOS after Ga1N intoxication also resulted in a significant improvement of GFR, but to lesser degree than in the case of treatment before intoxication. Therefore inhibition of the NO system before liver damage turned out to be more effective than inhibition after, although the differences were hardly significant.

In the literature there have been numerous attempts to define NO's role in HRS pathogenesis. But most of these studies used mainly a cirrhotic model of HRS [15, 28-34]. An increased concentration of CGMP, a second messenger of NO, was found in tissue homogenates of superior mesenteric arteries of portal hypertensive rats and also in vitro in a cell line coincubated with mesenteric arteries. Other studies showed that acute administration of NO inhibitor resulted in total normalization of splanchnic haemodynamics with reduction in portal venous inflow and increase in splanchnic vascular resistance in portal hypertensive rats. Moreover, in portal hypertension there is vascular hyporesponsiveness to different vasoconstrictors such as norepinephrine, angiotensin, vasopressin and endothelin. But the inhibition of NO biosynthesis restores vascular reactivity to normal levels, thus proving the importance and main role of NO in the regulation of the splanchnic vascular bed.

Our studies are probably the first in the available literature in which the acute galactosamine model of HRS is used to determine the role of the NO system in pathogenesis of this syndrome. However, the studies by Ros et al. [35] in cirrhotic rats showed that acute blockade of the NO system had no influence on renal blood flow, though it increased glomerular filtration. It was in contradiction to the studies by Martin et al. [36] in which they noted a lack of influence of NO inhibition on GFR level in cirrhotic rats. In our study, in an acute galactosamine model of HRS, we have shown that the blockade of NO synthesis results in a significant improvement of GFR, both before and after liver damage, and the pretreatment turned out to be more effective.

Regarding the activation of NO system, no attempt of its activation in our study before liver damage change significantly either level of GFR or concentration of creatinine and urea. However, using a donor for the NO system after Ga1N intoxication improved glomerular filtration, although not significantly. This problem was also raised in the literature, but only in the chronic cirrhotic model of HRS. Zhang et al. [37] and Kawada et al. [38] confirmed in their studies that mechanisms of activa- tion of endogenous NO system by using its donors completely abolished the vasoconstrictive effect of endothelin-1 on intrahepatic vessels and activated hepatic stellate cells in in vitro culture. But using NO donors in HRS chronic models creates some difficulties in in vivo studies. This phenomenon is mainly related to decreased number of sinusoidal endothelial cells and change of the histoarchitectonics in cirrhotic liver. Therefore, changed sinusoidal endothelial cells are unable to induce effective NO synthesis, even in at maximal blood flow and shear stress - factors releasing endogenous NO $[39,40]$. That is why we chose an acute model of HRS and intraperitoneal way of NO donor injection. But the results were different from those expected. We expected either a deterioration of renal efficiency or no changes in GFR. It appeared that the activation of NO synthesis could influence renal efficiency favourably. We think that in our acute experiment of Ga1N intoxication, there are no such histoarchitecture liver tissue changes, which are observed in cirrotic liver, which let endogenous NO be synthesized in a greater part of sinusoidal endothelial cells. In these conditions, using a donor for NO system showed its vasodilating activity in sinusoidal vessels, decreasing the intrahepatic vascular resitance and the pressure in the splanchnic vascular bed. This mechanisms may lead to increase of effective plasma volume and consequently to an improvement of renal efficiency [41].

Certainly, this observation requires further studies but we believe these results could confirm rather the hypothesis of the modulating property of L-arginine in NO synthesis than a simple activation of this biological system [42].

In conclusion, our study showed, that unlike Sprague-Dawley, Wistar rat model did not developed functional renal failure typical for hepatorenal syndrome. This is probably a first observation that some congenital, genetic predispositions might be responsible for the development of hepatorenal syndrome in experimental models. The inhibition of endogenous NO system both before and after liver damage results in an improvement of glomerular filtration. Pretreatment turned out to be more effective than treatment after liver intoxication. Attempting to activate of NO system before liver injury was without any influence on renal efficiency. However, activation of NO after Ga1N injection influenced favourably the level of glomerular filtration.

\footnotetext{
References

1. Wong F, Nadim MK, Kellum JA, et al. Working Party proposal for a revised classification system of renal dysfunction in patients with cirrhosis. Gut 2011; 60: 702-9.

2. Biecker E. Diagnosis and therapy of ascites in liver cirrhosis. World J Gastroenterol 2011; 17: 1237-48.

3. Cárdenas A, Ginès P. Acute-on-chronic liver failure: the kidneys. Curr Opin Crit Care 2011; 17: 184-9.
} 
4. Venkat D, Venkat KK. Hepatorenal syndrome. South Med J 2010; 103: 654-61.

5. Sussman AN, Boyer TD. Management of refractory ascites and hepatorenal syndrome. Curr Gastroenterol Rep 2011; 13: $17-25$.

6. Prakash J, Mahapatra AK, Ghosh B, Arora P, Jain AK. Clinical spectrum of renal disorders in patients with cirrhosis of liver. Ren Fail 2011; 33: 40-6.

7. Mazur JE, Cooper TB, Dasta JF. Terlipressin in hepatorenal syndrome. Ann Pharmacoter 2011; 45: 380-7.

8. Rahimi RS, Rockey DC. Complications and outcomes in chronic liver disease. Curr Opin Gastroenterol 2011; 27 . 204-9.

9. Kayali Z, Herring J, Baron P, et al. Increased plasma nitric oxide, L-arginine, and arginase- 1 in cirrhotic patients with progressive renal dysfunction. J Gastroenterol Hepatol 2009; 24: 1030-7.

10. Richir MC, Bouwman RH, Teerlink T, Siroen MP, de Vries $\mathrm{TP}$, van Leeuwen PA. The prominent role of the liver in the elimination of asymmetric dimethylarginine (ADMA) and the consequences of impaired hepatic function. JPEN J Parenter Enteral Nutr 2008; 32: 613-21.

11. Weerateerangkul P, Chattipakorn S, Chattipakorn N. Roles of the nitric oxide signaling pathway in cardiac ischemic preconditioning against myocardial ischemia-reperfusion injury. Med Sci Monit 2011; 17: RA44-52.

12. Sadeghi-Hashjin G, Abuhosseini M, Asri-Rezaei S. Role of nitric oxide in the plasma lipid profile in the rabbits. Arch Med Sci 2009; 4: 308-12.

13. Ytreb $\emptyset$ LM, Sen S, Rose C, et al. Systemic and regional hemodynamics in pigs with acute liver failure and the effect of albumin dialysis. Scand J Gastroenterol 2006; 41: 1350-60.

14. Rivera-Huizar S, Rincón-Sánchez AR, Covarrubias-Pinedo $A$, et al. Renal dysfunction as a consequence of acute liver damage by bile duct ligation in cirrhotic rats. Exp Toxicol Pathol 2006; 58: 185-95.

15. Islas-Carbajal MC, Covarrubias A, Grijalva G, Alvarez-Rodríguez A, Armendáriz-Borunda J, Rincón-Sánchez AR. Nitric oxide synthases inhibition results in renal failure improvement in cirrhotic rats. Liver Int 2005; 25: 131-40.

16. Martell M, Coll M, Ezkurdia N, Raurell I, Genescà J. Physiopathology of splanchnic vasodilation in portal hypertension. World J Hepatol 2010; 2: 208-20.

17. Regimbeau JM, Fuks D, Kohneh-Shahri N, Terris B, Soubrane $O$. Restrictive model of compensated carbon tetrachloride-induced cirrhosis in rats. World J Gastroentero 2008; 14: 6943-7.

18. Fujii T, Fuchs BC, Yamada S, et al. Mouse model of carbon tetrachloride induced liver fibrosis: Histopathological changes and expression of CD133 and epidermal growth factor. BMC Gastroenterol 2010; 10: 79.

19. Assimakopoulos SF, Vagianos CE. Bile duct ligation in rats: a reliable model of hepatorenal syndrome? World J Gastroenterol 2009; 15: 121-3.

20. Fu JL, Wang ZH, Li GZ, Wang YR, Liu P. Decreased IgA+ plasma cells and IgA expression in acute liver necrosis mice. World J Gastroenterol 2010; 16: 3827-33.

21. Tuñón MJ, Alvarez M, Culebras JM, González-Gallego J. An overview of animal models for investigating the pathogenesis and therapeutic strategies in acute hepatic failure. World J Gastroenterol 2009; 15: 3086-98.

22. Anand R, Harry D, Holt S, et al. Endothelin is an important determinant of renal function in a rat model of acute liver and renal failure. Gut 2002; 50: 111-7.

23. Javlé P, Yates J, Kynaston HG, Parsons KF, Jenkins SA Hepatosplanchnic haemodynamics and renal blood flow and function in rats with liver failure. Gut 1998; 43: 272-79.

24. Erdely A, Wagner L, Muller V, Szabo A, Baylis C. Protection of wistar furth rats from chronic renal disease is associ- ated with maintained renal nitric oxide synthase. J Am Soc Nephrol 2003; 14: 2526-33.

25. Erdely A, Freshour G, Tain YL, Engels K, Baylis C. DOCA/NaClinduced chronic kidney disease: a comparison of renal nitric oxide production in resistant and susceptible rat strains. Am J Physiol Renal Physiol 2007; 292: F192-6.

26. Erdely A, Freshour G, Baylis C. Resistance to renal damage by chronic nitric oxide synthase inhibition in the WistarFurth rat. Am J Physiol Regul Integr Comp Physiol 2006; 290: R66-72.

27. Erdely A, Freshour G, Smith C, Engels K, Olson JL, Baylis C. Protection against puromycin aminonucleoside-induced chronic renal disease in the Wistar-Furth rat. Am J Physiol Renal Physiol 2004; 287: F81-89.

28. Xu J, Cao H, Liu H, Wu ZY. Role of nitric oxide synthase and cyclooxygenase in hyperdynamic splanchnic circulation of portal hypertension. Hepatobiliary Pancreat Dis Int 2008; 7: 503-8.

29. Epstein M, Goligorsky MS. Endothelin and nitric oxide in hepatorenal syndrome: a balance reset. J Nephrol 1997; 10: $120-35$.

30. Biecker E, Trebicka J, Kang A, Hennenberg M, Sauerbruch T, Heller J. Treatment of bile duct-ligated rats with the nitric oxide synthase transcription enhancer AVE 9488 ameliorates portal hypertension. Liver Int 2008; 28: 331-8.

31. Anegawa G, Kawanaka H, Yoshida D, et al. Defective endothelial nitric oxide synthase signaling is mediated by rhokinase activation in rats with secondary biliary cirrhosis. Hepatology 2008; 47: 966-77.

32. Laleman W, Omasta A, Van de Casteele $M$, et al. A role for asymmetric dimethylarginine in the pathophysiology of portal hypertension in rats with biliary cirrhosis. Hepatology 2005; 42: 1382-90.

33. Stefano GB, Esch T, Bilfinger TV, Kream RM. Proinflammation and preconditioning protection are part of a common nitric oxide mediated process. Med Sci Monit 2010; 16: RA125-30.

34. Kream RM, Stefano GB. Interactive effects of endogenous morphine, nitric oxide, and ethanol on mitochondrial processes. Arch Med Sci 2010; 6: 658-62.

35. Ros J, Clària J, Jiménez W, et al. Role of nitric oxide and prostacyclin in the control of renal perfusion in experimental cirrhosis. Hepatology 1995; 22: 915-20.

36. Martin PY, Ginès P, Schrier RW. Nitric oxide as a mediator of hemodynamic abnormalities and sodium and water retention in cirrhosis. N Engl J Med 1998; 339: 533-41.

37. Zhang JX, Pegoli W, Clemens MG. Endothelin-1 induces direct constriction of hepatic sinusoids. Am J Physiol 1994; 226: G624-32.

38. Kawada N, Tran-Thi TA, Decker K. The contraction of hepatic stellate cells (Ito) stimulated with vasoactive substances. Possible involvement of endothelin 1 and nitric oxide in the regulation of sinusoidal tonus. Eur Biochem 1993; 213: 815-23.

39. Cardenas A. Hepatorenal syndrome: a decreased complication of end-stage liver disease. Am J Gastroenterol 2005; 100: 460-7.

40. Shah V, Lyford G, Gores G, Farrugia G. Nitric oxide in gastrointestinal health and disease. Gastroenterolgy 2004; 126: 903-13.

41. Alan C, Kocoglu H, Altintas R, Alici B, Ersay AR. Protective effect of decorin on acute ischaemia-reperfusion injury in the rat kidney. Arch Med Sci 2011; 7: 211-6.

42. Barylski M, Małyszko J, Rysz J, Myśliwiec M, Banach M. Lipids, blood pressure, kidney - what was new in 2011? Arch Med Sci 2011; 7: 1055-66. 